\title{
Quasipolynomial Computation of Nested Fixpoints
}

\author{
Daniel Hausmann (凶)(iD and Lutz Schröder $(\bowtie)$ (D) * \\ Friedrich-Alexander-Universität Erlangen-Nürnberg, Erlangen, Germany \\ $\{$ daniel.hausmann, lutz.schroeder $\} @ f a u . d e$
}

\begin{abstract}
It is well-known that the winning region of a parity game with $n$ nodes and $k$ priorities can be computed as a $k$-nested fixpoint of a suitable function; straightforward computation of this nested fixpoint requires $\mathcal{O}\left(n^{\frac{k}{2}}\right)$ iterations of the function. Calude et al.'s recent quasipolynomial-time parity game solving algorithm essentially shows how to compute the same fixpoint in only quasipolynomially many iterations by reducing parity games to quasipolynomially sized safety games. Universal graphs have been used to modularize this transformation of parity games to equivalent safety games that are obtained by combining the original game with a universal graph. We show that this approach naturally generalizes to the computation of solutions of systems of any fixpoint equations over finite lattices; hence, the solution of fixpoint equation systems can be computed by quasipolynomially many iterations of the equations. We present applications to modal fixpoint logics and games beyond relational semantics. For instance, the model checking problems for the energy $\mu$-calculus, finite latticed $\mu$-calculi, and the graded and the (two-valued) probabilistic $\mu$-calculus - with numbers coded in binary - can be solved via nested fixpoints of functions that differ substantially from the function for parity games but still can be computed in quasipolynomial time; our result hence implies that model checking for these $\mu$-calculi is in QP. Moreover, we improve the exponent in known exponential bounds on satisfiability checking.
\end{abstract}

Keywords: Fixpoint theory, model checking, satisfiability checking, parity games, energy games, $\mu$-calculus

\section{Introduction}

Fixpoints are pervasive in computer science, governing large portions of recursion theory, concurrency theory, logic, and game theory. One famous example are parity games, which are central, e.g., to networks and infinite processes [5], tree automata [43], and $\mu$-calculus model checking [17]. Winning regions in parity games can be expressed as nested fixpoints of particular set functions (e.g. $[8,16])$. In recent breakthrough work on the solution of parity games in quasipolynomial

\footnotetext{
* Work forms part of the DFG-funded project CoMoC (SCHR 1118/15-1, MI 717/7-1).

(C) The Author(s) 2021

J. F. Groote and K. G. Larsen (Eds.): TACAS 2021, LNCS 12651, pp. 38-56, 2021.

https://doi.org/10.1007/978-3-030-72016-2_3
} 
time, Calude et al. [9] essentially show how to compute this particular fixpoint in quasipolynomial time, that is, in time $2^{\mathcal{O}\left((\log n)^{c}\right)}$ for some constant $c$. Subsequently, it has been shown $[13,14,28]$ that universal graphs (that is, even graphs into which every even graph of a certain size embeds by a graph morphism) can be used to transform parity games to equivalent safety games obtained by pairing the original game with a universal graph; the size of these safety games is determined by the size of the employed universal graphs and it has been shown $[13,14]$ that there are universal graphs of quasipolynomial size. This yields a uniform algorithm for solving parity games to which all currently known quasipolynomial algorithms for parity games have been shown to instantiate using appropriately defined universal graphs $[13,14]$.

Briefly, our contribution in the present work is to show that the method of using universal graphs to solve parity games generalizes to the computation of nested fixpoints of arbitrary functions over finite lattices. That is, given functions $f_{i}: \mathcal{P}(U)^{k+1} \rightarrow \mathcal{P}(U), 0 \leq i \leq k$ on a finite lattice $U$, we give an algorithm that uses universal graphs to compute the solutions of systems of equations

$$
X_{i}={ }_{\eta_{i}} f_{i}\left(X_{0}, \ldots, X_{k}\right) \quad 0 \leq i \leq k
$$

where $\eta_{i}=$ GFP (greatest fixpoint) or $\eta_{i}=$ LFP (least fixpoint). Since there are universal graphs of quasipolynomial size, the algorithm requires only quasipolynomially many iterations of the functions $f_{i}$ and hence runs in quasipolynomial time, provided that all $f_{i}$ are computable in quasipolynomial time. While it seems plausible that this time bound may also be obtained by translating equation systems to equivalent standard parity games by emulating Turing machines to encode the functions $f_{i}$ as Boolean circuits (leading to many additional states but avoiding exponential blowup during the process), we emphasize that the main point of our result is not so much the ensuing time bound but rather the insight that universal graphs and hence many algorithms for parity games can be used on a much more general level which yields a precise (and relatively low) quasipolynomial bound on the number of function calls that are required to obtain solutions of fixpoint equation systems.

In more detail, the method of Calude et al. can be described as annotating nodes of a parity game with histories of quasipolynomial size and then solving this annotated game, but with a safety winning condition instead of the much more involved parity winning condition. It has been shown that these histories can be seen as nodes in universal graphs, in a more general reduction of parity games to safety games in which nodes from the parity game are annotated with nodes from a universal graph. This method has also been described as pairing separating automata with safety games [14]. It has been shown $[13,14]$ that there are exponentially sized universal graphs (essentially yielding the basis for e.g. the fixpoint iteration algorithm [8] or the small progress measures algorithm [27]) and quasipolynomially sized universal graphs (corresponding, e.g., to the succinct progress measure algorithm [28], or to the recent quasipolynomial variant of Zielonka's algorithm [38]).

Hasuo et al. [22], and more generally, Baldan et al. [4] show that nested fixpoints in highly general settings can be computed by a technique based on 
progress measures, implicitly using exponentially sized universal graphs, obtaining an exponential bound on the number of iterations. Our technique is based on showing that one can make explicit use of universal graphs, correspondingly obtaining a quasipolynomial upper bound on the number of iterations. In both cases, computation of the nested fixpoint is reduced to a single (least or greatest depending on exact formulation) fixpoint of a function that extends the given set function to keep track of the exponential and quasipolynomial histories, respectively, in analogy to the previous reduction of parity games to safety games. Our central result can then be phrased as saying that the method of transforming parity conditions to safety conditions using universal graphs generalizes from solving parity games to solving systems of equations that use arbitrary functions over finite lattices. We use fixpoint games $[4,42]$ to obtain the crucial result that the solutions of equation systems have history-free witnesses, in analogy to history-freeness of winning strategies in parity games. These fixpoint games have exponential size but we show how to extract polynomial-size witnesses for winning strategies of Eloise, and use these witnesses to show that any node won by Eloise is also won in the safety game obtained by a universal graph. For the backwards direction, we show that a witness for satisfaction of the safety condition regarding the universal graph induces a winning strategy in the fixpoint game. This proves that universal graphs can be used to compute nested fixpoints of arbitrary functions over finite lattices and hence yields the quasipolynomial upper bound for computation of nested fixpoints. Moreover, we present a progress measure algorithm that uses the nodes of a quasipolynomial universal graph to measure progress and that can be used to efficiently compute nested fixpoints of arbitrary functions over finite lattices.

As an immediate application of these results, we improve known deterministic algorithms for solving energy parity games [10], that is, parity games in which edges have additional integer weights and for which the winning condition is a combined parity condition and a (quantitative) positivity condition on the sum of the accumulated weights. Our results also show that the model checking problem for the associated energy $\mu$-calculus [2] is in QP. In a similar fashion, we obtain quasipolynomial algorithms for model checking in latticed $\mu$-calculi [7] in which the truth values of formulae are computed over arbitrary finite lattices, and for solving associated latticed parity games [30].

Furthermore, our results improve generic upper complexity bounds on model checking and satisfiability checking in the coalgebraic $\mu$-calculus [12], which serves as a generic framework for fixpoint logics beyond relational semantics. Well-known instances of the coalgebraic $\mu$-calculus include the alternatingtime $\mu$-calculus [1], the graded $\mu$-calculus [32], the (two-valued) probabilistic $\mu$-calculus [12,34], and the monotone $\mu$-calculus [18] (the ambient fixpoint logic of concurrent dynamic logic CPDL [39] and Parikh's game logic [37]). This level of generality is achieved by abstracting system types as set functors and systems as coalgebras for the given functor following the paradigm of universal coalgebra [40]. It was previously shown [24] that the model checking problem for coalgebraic $\mu$-calculi reduces to the computation of a nested fixpoint. This 
fixpoint may be seen as a coalgebraic generalization of a parity game winning region but can be literally phrased in terms of small standard parity games (implying quasipolynomial run time) only in restricted cases. Our results show that the relevant nested fixpoint can be computed in quasipolynomial time in all cases of interest. Notably, we thus obtain as new specific upper bounds that even under binary coding of numbers, the model checking problems of both the graded $\mu$-calculus and the probabilistic $\mu$-calculus are in QP, even when the syntax is extended to allow for (monotone) polynomial inequalities.

Similarly, the satisfiability problem of the coalgebraic $\mu$-calculus has been reduced to a computation of a nested fixpoint [25], and our present results imply a marked improvement in the exponent of the associated exponential time bound. Specifically, the nesting depth of the relevant fixpoint is exponentially smaller than the basis of the lattice. Our results imply that this fixpoint is computable in polynomial time so that the complexity of satisfiability checking in coalgebraic $\mu$-calculi drops from $2^{\mathcal{O}\left(n^{2} k^{2} \log n\right)}$ to $2^{\mathcal{O}(n k \log n)}$ for formulae of size $n$ and with alternation depth $k$.

Related Work The quasipolynomial bound on parity game solving has in the meantime been realized by a number of alternative algorithms. For instance, Jurdzinski and Lazic [28] use succinct progress measures to improve to quasilinear (instead of quasipolynomial) space; Fearnley et al. [19] similarly achieve quasilinear space. Lehtinen [33] and Boker and Lehtinen [6] present a quasipolynomial algorithm using register games. Parys [38] improves Zielonka's algorithm [43] to run in quasipolynomial time. In particular the last algorithm is of interest as an additional candidate for generalization to nested fixpoints, due to the known good performance of Zielonka's algorithm in practice. Daviaud et al. [15] generalize quasipolynomial-time parity game solving by providing a pseudoquasipolynomial algorithm for mean-payoff parity games. On the other hand, Czerwinski et al. [14] give a quasipolynomial lower bound on universal trees, implying a barrier for prospective polynomial-time parity game solving algorithms. Chatterjee et al. [11] describe a quasipolynomial time set-based symbolic algorithm for parity game solving that is parametric in a lift function that determines how ranks of nodes depend on the ranks of their successors, and thereby unifies the complexity and correctness analysis of various parity game algorithms. Although part of the parity game structure is encapsulated in a set operator CPre, the development is tied to standard parity games, e.g. in the definition of the best function, which picks minimal or maximal ranks of successors depending on whether a node belongs to Abelard or Eloise.

Early work on the computation of unrestricted nested fixpoints has shown that greatest fixpoints require less effort in the fixpoint iteration algorithm, which can hence be optimized to compute nested fixpoints with just $\mathcal{O}\left(n^{\frac{k}{2}}\right)$ calls of the functions at hand [35,41], improving the previously known (straightforward) bound $\mathcal{O}\left(n^{k}\right)$; here, $n$ denotes the size of the basis of the lattice and $k$ the number of fixpoint operators. Recent progress in the field has established the abovementioned approaches using progress measures [22] and fixpoint games [4] in general settings, both with a view to applications in coalgebraic model checking 
like in the present paper. In comparison to the present work, the respective bounds on the required number of function iterations in the above unrestricted approaches all are exponential.

A preprint of our present results, specifically the quasipolynomial upper bound on function iteration in fixpoint computation, has been available as an arXiv preprint for some time [23]. Subsequent to this preprint, Arnold, Niwinski and Parys [3] have improved the actual run time by reducing the overhead incurred per iteration (and they give a form of quasipolynomial lower bound for universal-tree-based algorithms), working (like [23]) in the less general setting of directly nested fixpoints over powerset lattices; we show in Section 6 how such an improvement can be incorporated also in our lattice-based algorithm.

\section{Notation and Preliminaries}

Let $U$ and $V$ be sets, and let $R \subseteq U \times U$ be a binary relation on $U$. For $u \in U$, we then put $R(u):=\{v \in U \mid(u, v) \in R\}$. We put $[k]=\{0, \ldots, k\}$ for $k \in \mathbb{N}$. Labelled graphs $G=(W, R)$ consist of a set $W$ together with a relation $R \subseteq W \times A \times W$ where $A$ is some set of labels; typically, we use $A=[k]$ for some $k \in \mathbb{N}$. An $R$-path in a labelled graph is a finite or infinite sequence $v_{0}, a_{0}, v_{1}, a_{1}, v_{2} \ldots$ (ending in a node from $W$ if finite) such that $\left(v_{i}, a_{i}, v_{i+1}\right) \in R$ for all $i$. For $v \in W$ and $a \in A$, we put $R_{a}(v)=\{w \in W \mid(v, a, w) \in R\}$ and sometimes write $|G|$ to refer to $|W|$. As usual, we write $U^{*}$ and $U^{\omega}$ for the sets of finite sequences or infinite sequences, respectively, of elements of $U$. The domain $\operatorname{dom}(f)$ of a partial function $f: U \rightarrow V$ is the set of elements on which $f$ is defined. As usual, the (forward) image of $A^{\prime} \subseteq A$ under a function $f: A \rightarrow B$ is $f\left[A^{\prime}\right]=\left\{b \in B \mid \exists a \in A^{\prime} . f(a)=b\right\}$ and the preimage $f^{-1}\left[B^{\prime}\right]$ of $B^{\prime} \subseteq B$ under $f$ is defined by $f^{-1}\left[B^{\prime}\right]=\left\{a \in A \mid \exists b \in B^{\prime} \cdot f(a)=b\right\}$. Projections $\pi_{j}: A_{1} \times \ldots \times A_{m} \rightarrow A_{j}$ for $1 \leq j \leq m$ are given by $\pi_{i}\left(a_{1}, \ldots, a_{m}\right)=a_{j}$. We often regard (finite) sequences $\tau=u_{0}, u_{1}, \ldots \in U^{*} \cup U^{\omega}$ of elements of $U$ as partial functions of type $\mathbb{N} \rightarrow U$ and then write $\tau(i)$ to denote the element $u_{i}$, for $i \in \operatorname{dom}(\tau)$. For $\tau \in U^{*} \cup U^{\omega}$, we define the set $\operatorname{lnf}(\tau)=\{u \in U \mid \forall i \geq$ $0 . \exists j>i . \tau(j)=u\}$ of elements that occur infinitely often in $\tau(\operatorname{so} \operatorname{lnf}(\tau)=\emptyset$ for $\left.\tau \in U^{*}\right)$. An infinite $R$-path $v_{0}, p_{0}, v_{1}, p_{1}, \ldots$ in a labelled graph $G=(W, R)$ with labels from $[k]$ is even if $\max \left(\operatorname{lnf}\left(p_{0}, p_{1}, \ldots\right)\right)$ is even, and $G$ is even if every infinite $R$-path in $G$ is even. We write $\mathcal{P}(U)$ for the powerset of $U$, and $U^{m}$ for the $m$-fold Cartesian product $U \times \cdots \times U$.

Finite Lattices and Fixpoints A finite lattice $(L, \sqsubseteq)$ (often written just as $L$ ) consists of a non-empty finite set $L$ together with a partial order $\sqsubseteq$ on $L$, such that there is, for all subsets $X \subseteq L$, a join $\bigsqcup X$ and a meet $\Pi X$. The least and greatest elements of $L$ are defined as $\top=\bigsqcup \emptyset$ and element $\top=\emptyset \emptyset$, respectively. A set $B_{L} \subseteq L$ such that $l=\bigsqcup\left\{b \in B_{L} \mid b \sqsubseteq l\right\}$ is a basis of $L$. Given a finite lattice $L$, a function $g: L^{k} \rightarrow L$ is monotone if $g\left(V_{1}, \ldots, V_{k}\right) \sqsubseteq g\left(W_{1}, \ldots, W_{k}\right)$ whenever $V_{i} \sqsubseteq W_{i}$ for all $1 \leq i \leq k$. For monotone $f: L \rightarrow L$, we put

$$
\text { GFP } f=\bigsqcup\{V \sqsubseteq L \mid V \sqsubseteq f(V)\} \quad \operatorname{LFP} f=\prod\{V \sqsubseteq L \mid f(V) \sqsubseteq V\},
$$


which, by the Knaster-Tarski fixpoint theorem, are the greatest and the least fixpoint of $f$, respectively. Furthermore, we define $f^{0}(V)=V$ and $f^{m+1}(V)=$ $f\left(f^{m}(V)\right)$ for $m \geq 0, V \sqsubseteq L$; since $L$ is finite, we have GFP $f=f^{n}(T)$ and LFP $f=f^{n}(\perp)$ by Kleene's fixpoint theorem. Given a finite set $U$ and a natural number $n,\left(n^{U}, \sqsubseteq\right)$ is a finite lattice, where $n^{U}=\{f: U \rightarrow[n-1]\}$ denotes the function space from $U$ to $[n-1]$ and $f \sqsubseteq g$ if and only if for all $u \in U, f(u) \leq g(u)$. For $n=2$, we obtain the powerset lattice $\left(2^{U}, \subseteq\right)$, also denoted by $\mathcal{P}(U)$, with least and greatest elements $\emptyset$ and $U$, respectively, and basis $\{\{u\} \mid u \in U\}$.

Parity games A parity game $(V, E, \Omega)$ consists of a set of nodes $V$, a left-total relation $E \subseteq V \times V$ of moves encoding the rules of the game, and a priority function $\Omega: V \rightarrow \mathbb{N}$, which assigns priorities $\Omega(v) \in \mathbb{N}$ to nodes $v \in V$. Moreover, each node belongs to exactly one of the two players Eloise or Abelard, where we denote the set of Eloise's nodes by $V_{\exists}$ and that of Abelard's nodes by $V_{\forall}$. A play $\rho \in V^{\omega}$ is an infinite sequence of nodes that follows the rules of the game, that is, such that for all $i \geq 0$, we have $(\rho(i), \rho(i+1)) \in E$. We say that an infinite play $\rho=v_{0}, v_{1}, \ldots$ is even if the largest priority that occurs infinitely often in it (i.e. $\max (\operatorname{lnf}(\Omega \circ \rho)))$ is even, and odd otherwise, and call this property the parity of $\rho$. Player Eloise wins exactly the even plays and player Abelard wins all other plays. A (history-free) Eloise-strategy $s: V_{\exists} \rightarrow V$ is a partial function that assigns single moves $s(x)$ to Eloise-nodes $x \in \operatorname{dom}(s)$. Given an Eloise-strategy $s$, a play $\rho$ is an $s$-play if for all $i \in \operatorname{dom}(\rho)$ such that $\rho(i) \in V_{\exists}$, we have $\rho(i+1)=s(\rho(i))$. An Eloise-strategy wins a node $v \in V$ if Eloise wins all $s$-plays that start at $v$. We have a dual notion of Abelard-strategies; solving a parity game consists in computing the winning regions $\operatorname{win}_{\exists}$ and $\operatorname{win}_{\forall}$ of the two players, that is, the sets of states that they respectively win by some strategy.

It is known that solving parity games is in $\mathrm{NP} \cap \mathrm{CONP}$ (and, more specifically, in UP $\cap \mathrm{CO}-\mathrm{UP}$ ). Recently it has also been shown [9] that for parity games with $n$ nodes and $k$ priorities, $\operatorname{win}_{\exists}$ and $\operatorname{win}_{\forall}$ can be computed in quasipolynomial time $\mathcal{O}\left(n^{\log k+6}\right)$. Another crucial property of parity games is that they are history-free determined [21], that is, that every node in a parity game is won by exactly one of the two players and then there is a history-free strategy for the respective player that wins the node.

\section{Systems of Fixpoint Equations}

We now introduce our central notion, that is, systems of fixpoint equations over a finite lattice. Throughout, we fix a finite lattice $(L, \sqsubseteq)$ and a basis $B_{L}$ of $L$ such that $\perp \notin B_{L}$, and $k+1$ monotone functions $f_{i}: L^{k+1} \rightarrow L, 0 \leq i \leq k$.

Definition 3.1. A system of equations consists of $k+1$ equations of the form

$$
X_{i}={ }_{\eta_{i}} f_{i}\left(X_{0}, \ldots, X_{k}\right)
$$


where $\eta_{i} \in\{$ LFP, GFP $\}$, briefly referred to as $f$. For a partial valuation $\sigma:[k] \rightarrow$ $L$, we inductively define

$$
\llbracket X_{i} \rrbracket^{\sigma}=\eta_{i} X_{i} \cdot f_{i}^{\sigma}
$$

where the function $f_{i}^{\sigma}$ is given by

$$
f_{i}^{\sigma}(A)=f_{i}\left(\llbracket X_{0} \rrbracket^{\sigma^{\prime}}, \ldots, \llbracket X_{i-1} \rrbracket^{\sigma^{\prime}}, A, \operatorname{ev}\left(\sigma^{\prime}, i+1\right), \ldots, \mathrm{ev}\left(\sigma^{\prime}, k\right)\right)
$$

for $A \in L$, where $(\sigma[i \mapsto A])(j)=\sigma(j)$ for $j \neq i$ and $(\sigma[i \mapsto A])(i)=A$, $\sigma^{\prime}=\sigma[i \mapsto A]$ and where $\operatorname{ev}(\sigma, j)=\sigma(j)$ if $j \in \operatorname{dom}(\sigma)$ and $\operatorname{ev}(\sigma, j)=\llbracket X_{j} \rrbracket^{\sigma}$ otherwise (the latter clause handles free variables). Then, the solution of the system of equations is $\llbracket X_{k} \rrbracket^{\epsilon}$ where $\epsilon:[k] \rightarrow L$ denotes the empty valuation (i.e. $\operatorname{dom}(\epsilon)=\emptyset$ ). Similarly, we can obtain solutions for the other components as $\llbracket X_{i} \rrbracket^{\epsilon}$ for $0 \leq i<k$; we drop the valuation index if no confusion arises, and sometimes write $\llbracket X_{i} \rrbracket_{f}$ to make the equation system $f$ explicit. We denote by $\mathrm{E}^{f_{0}}$ the solution $\llbracket X_{k} \rrbracket$ for the canonical system of equations of the particular shape

$$
X_{i}={ }_{\eta_{i}} X_{i-1} \quad X_{0}={ }_{\text {GFP }} f_{0}\left(X_{0}, \ldots, X_{k}\right),
$$

where $0<i \leq k, \eta_{i}=\mathrm{LFP}$ for odd $i$ and $\eta_{i}=$ GFP for even $i$.

Example 3.2. (1) Parity games and the modal $\mu$-calculus: Let $(V, E, \Omega)$ be a parity game with priorities 0 to $k$, take $L=\mathcal{P}(V)$, and consider the canonical system of fixpoint equations $\mathrm{E}^{f_{\exists}}$ for the function $f_{\exists}: \mathcal{P}(V)^{k+1} \rightarrow \mathcal{P}(V)$ given by

$$
f_{\exists}\left(V_{0}, \ldots, V_{k}\right)=\left\{v \in V_{\exists} \mid E(v) \cap V_{\Omega(v)} \neq \emptyset\right\} \cup\left\{v \in V_{\forall} \mid E(v) \subseteq V_{\Omega(v)},\right\}
$$

for $\left(V_{0}, \ldots, V_{k}\right) \in \mathcal{P}(V)^{k+1}$. It is well known that win $_{\exists}=\mathrm{E}^{f_{\exists}}$, i.e. parity games can be solved by solving fixpoint equation systems. Intuitively, $v \in f_{\exists}\left(V_{0}, \ldots, V_{k}\right)$ iff Eloise can enforce that some node in $V_{\Omega(v)}$ is reached in the next step. The nested fixpoint expressed by $\mathrm{E}^{f_{\exists}}$ (in which least (greatest) fixpoints correspond to odd (even) priorities) is constructed in such a way that Eloise only has to rely infinitely often on an argument $V_{i}$ for odd $i$ if she can also ensure that some argument $V_{j}$ for $j>i$ is used infinitely often.

Model checking for the modal $\mu$-calculus [29] and solving parity games are linear-time equivalent problems. Formulae of the $\mu$-calculus are evaluated over Kripke frames $(U, R)$ with set of states $U$ and transition relation $R$. Formulae $\phi$ of the $\mu$-calculus can be directly represented as equation systems over the lattice $\mathcal{P}(U)$ by recursively translating $\phi$ to equations, mapping subformulae $\mu X_{i} . \psi\left(X_{0}, \ldots, X_{k}\right)$ and $\nu X_{j} . \psi\left(X_{0}, \ldots, X_{k}\right)$ to equations

$$
X_{i}={ }_{\mu} \psi\left(X_{0}, \ldots, X_{k}\right) \quad X_{j}={ }_{\nu} \chi\left(X_{0}, \ldots, X_{k}\right),
$$

and interpreting the modalities $\diamond$ and $\square$ by functions

$$
f_{\diamond}(X)=\{u \in U \mid R(u) \cap X \neq \emptyset\} \quad f_{\square}(X)=\{u \in U \mid R(u) \subseteq X\}
$$


The solution of the resulting system of equations then is the truth set of the formula $\phi$, that is, model checking for the model $\mu$-calculus reduces to solving fixpoint equation systems. Furthermore, satisfiability checking for the modal $\mu$ calculus can be reduced to solving so-called satisfiability games [20], that is, parity games that are played over the set of states of a determinized parity automaton. These satisfiability games can be expressed as systems of fixpoint equations, where the functions track transitions in the determinized automaton.

(2) Energy parity games and the energy $\mu$-calculus: Energy parity games [10] are two-player games played over weighted game arenas $(V, E, w, \Omega)$, where $w: E \rightarrow$ $\mathbb{Z}$ assigns integer weights to edges. The winning condition is the combination of a parity condition with a (quantitative) positivity condition on the sum of the accumulated weights. It has been shown $[2,10]$, that $b=n \cdot d \cdot W$ is a sufficient upper bound on energy level accumulations in energy parity games with $n$ nodes, $k$ priorities and maximum absolute weight $W$. We define a function $f_{\exists}^{\mathrm{e}}:\left((b+1)^{V}\right)^{k+1} \rightarrow(b+1)^{V}$ over the finite lattice $(b+1)^{V}$ (whose elements are functions from $V$ to the set $\{0, \ldots, b+1\})$ by putting

$$
\left(f_{\exists}^{\mathrm{e}}\left(V_{0}, \ldots, V_{k}\right)\right)(v)= \begin{cases}\min \left(\operatorname{en}\left(v, V_{\Omega(v)}\right)\right) & \text { if } v \in V_{\exists} \\ \max \left(\operatorname{en}\left(v, V_{\Omega(v)}\right)\right) & \text { if } v \in V_{\forall}\end{cases}
$$

for $\left(V_{0}, \ldots, V_{k}\right) \in\left((b+1)^{V}\right)^{k+1}$ and $v \in V$, using en $(v, \sigma)$ as abbreviation for

$$
\begin{aligned}
\operatorname{en}(v, \sigma)= & \{n \in\{0, \ldots, b\} \mid \exists u \in E(v) . n=\max \{0, \sigma(u)-w(v, u)\}\} \cup \\
& \{b+1 \mid \exists u \in E(v) . \sigma(u)-w(v, u)>b \text { or } \sigma(u)>b\},
\end{aligned}
$$

where $\sigma: V \rightarrow\{0, \ldots, b+1\}$. Then it follows from the results of [2] that player Eloise wins a node $v$ in the energy parity game with minimal initial credit $c<b+1$ if $\left(\mathrm{E}_{\exists}^{f_{\exists}^{\mathrm{j}}}\right)(v)=c$, that is, if the solution of the canonical equation system over $f_{\exists}^{\mathrm{e}}$ maps $v$ to a value $c$ that is at most $b$.

The energy $\mu$-calculus [2] is the fixpoint logic that corresponds to energy parity games. Its formulae are evaluated over weighted game structures and involve operators $\nabla_{E} \phi$ and $\square_{E} \phi$ that are evaluated depending on the energy function $\llbracket \phi \rrbracket: V \rightarrow\{0, \ldots, b+1\}$ that is obtained by first evaluating the argument formula $\phi$. The semantics of the diamond operator then is an energy function that assigns, to each state $v$, the least energy value $c \in\{0, \ldots, b+1\}$ such that there is a move from $v$ to some node $u$ such that the credit $c$ suffices to take the move from $v$ to $u$ and retain an energy level of at least $\llbracket \phi \rrbracket(u)$. Formulae can be translated to equation systems over the finite lattice $(b+1)^{V}$, where the functions for modal operators are defined according to their semantics as presented in [2]. Solving these equation systems then amounts to model checking energy $\mu$-calculus formulae over weighted game structures.

(3) Latticed $\mu$-calculi: In latticed $\mu$-calculi [7], formulae are evaluated over complete lattices $L$ rather than the powerset lattice; for finite lattices $L$, formulae of latticed $\mu$-calculi hence can be translated to fixpoint equation systems over $L$, so that model checking reduces to solving equation systems. An associated latticed 
variant of games has been introduced in [30] and for finite lattices $L$, solving latticed parity games over $L$ reduces to solving equation systems over $L$.

(4) The coalgebraic $\mu$-calculus and coalgebraic parity games: The coalgebraic $\mu$-calculus [12] supports generalized modal branching types by using predicate liftings to interpret formulae over $T$-coalgebras, that is, over structures whose transition type is specified by an endofunctor $T$ on the category of sets. For instance the functors $T=\mathcal{P}, T=\mathcal{D}$ and $T=\mathcal{G}$ map sets $X$ to their powerset $\mathcal{P}(X)$, the set of probability distributions $\mathcal{D}(X)=\{f: X \rightarrow[0, \ldots, 1]\}$ over $X$, and to the set of multisets $\mathcal{G}(X)=\{f: X \rightarrow \mathbb{N}\}$ over $X$, respectively. The corresponding $T$-coalgebras then are Kripke frames (for $T=\mathcal{P}$ ), Markov chains (for $T=\mathcal{D}$ ) and graded transition systems (for $T=\mathcal{G}$ ), respectively. Instances of the coalgebraic $\mu$-calculus comprise, e.g. the two-valued probabilistic $\mu$-calculus $[12,34]$ with modalities $\nabla_{p} \phi$ for $p \in[0, \ldots, 1]$, expressing 'the next state satisfies $\phi$ with probability more than $p$; the graded $\mu$-calculus [32] with modalities $\nabla_{g} \phi$ for $g \in \mathbb{N}$, expressing 'there are more than $\phi$ successor states that satisfy $\phi$ '; or the alternating-time $\mu$-calculus [1] that is interpreted over concurrent game frames and uses modalities $\langle D\rangle \phi$ for finite $D \subseteq \mathbb{N}$ (encoding a coalition) that express that 'coalition $D$ has a joint strategy to enforce $\phi$ '.

It has been shown in previous work [24] that model checking for coalgebraic $\mu$-calculi against coalgebras with state space $U$ reduces to solving a canonical fixpoint equation system over the powerset lattice $\mathcal{P}(U)$, where the involved function interprets modal operators using predicate liftings, as described in [12,24]. This canonical equation system can alternatively be seen as the winning region of Eloise in coalgebraic parity games, a highly general variant of parity games where the game structure is a coalgebra and nodes are annotated with modalities. Examples include two-valued probabilistic parity games and graded parity games in which nodes and edges are annotated with probabilities or grades, respectively. In order to win a node $v$, player Eloise then has to have a strategy that picks a set of moves to nodes that in turn are all won by Eloise, and such that the joint probability (joint grade) of the picked moves is greater than the probability (grade) that is assigned to $v$. It is known that solving coalgebraic parity games reduces to solving fixpoint equation systems [24].

Furthermore, the satisfiability problem of the coalgebraic $\mu$-calculus has been reduced to solving canonical fixpoint equations systems over lattices $\mathcal{P}(U)$, where $U$ is the state set of a determinized parity automaton and where the innermost equation checks for joint one-step satisfiability of sets of coalgebraic modalities [25]. By interpreting coalgebraic formulae over finite lattices $d^{U}$ rather than over powerset lattices, one obtains the finite-valued coalgebraic $\mu$-calculus (with values $\{0, \ldots, d\}$ ), which has the finite-valued probabilistic $\mu$-calculus (e.g. [36]) as an instance. Model checking for the finite-valued probabilistic $\mu$-calculus hence reduces to solving equation systems over the finite lattice $d^{|U|}$, where $\{0, \ldots, d\}$ encodes a finite set of probabilities. 


\section{Fixpoint Games and History-free Witnesses}

We instantiate the existing notion of fixpoint games [4, 42], which characterize solutions of equation systems, to our setting (that is, to finite lattices), and then use these games as a technical tool to establish our crucial notion of historyfreeness for systems of fixpoint equations.

Definition 4.1 (Fixpoint games). Let $X_{i}={ }_{\eta_{i}} f_{i}\left(X_{0}, \ldots, X_{k}\right), 0 \leq i \leq k$, be a system of fixpoint equations. The associated fixpoint game is a parity game $(V, E, \Omega)$ with set of nodes $V=\left(B_{L} \times[k]\right) \cup L^{k+1}$, where nodes from $B_{L} \times[k]$ belong to player Eloise and nodes from $L^{k+1}$ belong to player Abelard. For nodes $(u, i) \in B_{L} \times[k]$, we put

$$
E(u, i)=\left\{\left(U_{0}, \ldots, U_{k}\right) \in L^{k+1} \mid u \sqsubseteq f_{i}\left(U_{0}, \ldots, U_{k}\right)\right\},
$$

and for nodes $\left(U_{0}, \ldots, U_{k}\right) \in L^{k+1}$, we put

$$
E\left(U_{0}, \ldots, U_{k}\right)=\left\{(u, i) \in B_{L} \times[k] \mid u \sqsubseteq U_{i}\right\} .
$$

The alternation depth ad $(i)$ of an equation $X_{i}={ }_{\eta_{i}} f_{i}\left(X_{0}, \ldots, X_{1}\right)$ is defined as $\operatorname{ad}_{i}^{\mu}$ if $\eta_{i}=\mu$ and as $\operatorname{ad}_{i}^{\nu}$ if $\eta_{i}=\nu$, where $\operatorname{ad}_{i}^{\mu}, \operatorname{ad}_{i}^{\nu}$ are recursively defined by

$$
\operatorname{ad}_{i}^{\mu}=\left\{\begin{array}{ll}
\operatorname{ad}_{i-1}^{\mu} & i>0, \eta_{i-1}=\mu \\
\operatorname{ad}_{i-1}^{\nu}+1 & i>0, \eta_{i-1}=\nu \\
1 & i=0
\end{array} \quad \operatorname{ad}_{i}^{\nu}= \begin{cases}\operatorname{ad}_{i-1}^{\mu}+1 & i>0, \eta_{i-1}=\mu \\
\operatorname{ad}_{i-1}^{\nu} & i>0, \eta_{i-1}=\nu \\
0 & i=0\end{cases}\right.
$$

for $0 \leq i \leq k$. The priority function $\Omega: V \rightarrow[\operatorname{ad}(k)]$ then is defined by $\Omega(u, i)=$ $\operatorname{ad}(i)$ and $\Omega\left(U_{0}, \ldots, U_{k}\right)=0$.

Remark 4.2. In [4], an alternative priority function $\Omega^{\prime}: V \rightarrow[2 k+1]$ with

$$
\Omega^{\prime}(u, i)= \begin{cases}2 i & \text { if } \eta_{i}=\mathrm{GFP} \\ 2 i+1 & \text { if } \eta_{i}=\mathrm{LFP}\end{cases}
$$

and $\Omega^{\prime}\left(U_{0}, \ldots, U_{k}\right)=0$ is used. Since $\operatorname{ad}(i)$ is even if and only if $\eta_{i}$ is even, and moreover $\operatorname{ad}(\mathrm{i}) \leq \operatorname{ad}(j)$ for $i \leq j$, and $i<j$ whenever $\operatorname{ad}(\mathrm{i})<\operatorname{ad}(j)$, it is easy to see that $\Omega$ and $\Omega^{\prime}$ in fact assign identical parities to all plays. In the following, we will use the more economic parity function $\Omega$ so that fixpoint games have only $d:=\operatorname{ad}(k) \leq k$ priorities.

We import the associated characterization theorem [4, Theorem 4.8]:

Theorem 4.3 ([4]). We have $u \sqsubseteq \llbracket X_{i} \rrbracket_{f}$ if and only if Eloise wins the node $(u, i)$ in the fixpoint game for the given system $f$ of equations.

Remark 4.4. While this shows that parity game solving can be used to solve equation systems, the size of fixpoint games is exponential in $\left|B_{L}\right|$, so they do not directly yield a quasipolynomial algorithm for solving equation systems. 
Next we define our notion of history-freeness for systems of fixpoint equations.

Definition 4.5 (History-free witness). A history-free witness for $u \sqsubseteq \llbracket X_{i} \rrbracket_{f}$ is an even labelled graph $(W, R)$ with labels from $[d]$ such that $W \subseteq B_{L} \times$ $[d],(u, i) \in W$, and for all $(v, p) \in W$, we have $v \sqsubseteq f_{p}\left(U_{0}, \ldots, U_{k}\right)$ where $U_{j}=\bigsqcup \pi_{1}\left[R_{\mathrm{ad}(j)}(v, p)\right]$ for $0 \leq j \leq k$, noting that $R_{\mathrm{ad}(j)}(v, p) \subseteq W$ so that $\pi_{1}\left[R_{\mathrm{ad}(j)}(v, p)\right] \subseteq B_{L}$ and $U_{j} \in L$.

In analogy to history-free strategies for parity games, history-free witnesses assign tuples $\left(R_{1}(v, p), \ldots, R_{d}(v, p)\right)$ of sets $R_{j}(v, p) \subseteq W$ to pairs $(v, p) \in W$ without relying on a history of previously visited pairs. We have $|W| \leq(d+1)\left|B_{L}\right|$ and $|R| \leq(d+1)|W|^{2}$, that is, the size of history-free witnesses is polynomial in $\left|B_{L}\right|$. Crucially, history-free witnesses always exist:

Lemma 4.6. For all $u \in B_{L}$ and $i \in[k]$, we have

$u \sqsubseteq \llbracket X_{i} \rrbracket_{f}$ if and only if there is a history-free witness for $u \sqsubseteq \llbracket X_{i} \rrbracket_{f}$.

Proof. In one direction, we have $u \sqsubseteq \llbracket X_{i} \rrbracket_{f}$ so that Eloise wins the node $(u, i)$ in the according fixpoint game by Lemma 4.3. Let $s$ be a corresponding historyfree winning strategy (such strategies always exists, see e.g. [21]). We inductively construct a witness for $u \sqsubseteq \llbracket X_{i} \rrbracket_{f}$, starting at $(u, i)$. When at $(v, p) \in B_{L} \times[k]$ with $s(v, p)=\left(U_{0}, \ldots, U_{k}\right)$, we put $R_{i}(v, p)=\bigcup_{j \mid \operatorname{ad}(j)=i}\left(U_{j} \times\{j\}\right)$ for $0 \leq i \leq d$ and hence have $\operatorname{ad}(j)=i$ for all $((v, p), i,(u, j)) \in R$. Since $s$ is a winning strategy, the resulting graph $(W, R)$ is a history-free witness for $u \sqsubseteq \llbracket X_{i} \rrbracket_{f}$ by construction; in particular, $(W, R)$ is even. For the converse direction, the witness for $u \sqsubseteq \llbracket X_{i} \rrbracket_{f}$ directly yields a winning Eloise-strategy for the node $(u, i)$ in the associated fixpoint game. This implies $u \sqsubseteq \llbracket X_{i} \rrbracket_{f}$ by Lemma 4.3.

\section{Solving Equation Systems using Universal Graphs}

We go on to prove our main result. To this end, we fix a system $f$ of fixpoint equations $f_{i}: L^{k+1} \rightarrow L, 0 \leq i \leq k$, and put $n:=\left|B_{L}\right|$ and $d:=\operatorname{ad}(k)$ for the remainder of the paper.

Definition 5.1 (Universal graphs $[13,14])$. Let $G=(W, R)$ and $G^{\prime}=$ $\left(W^{\prime}, R^{\prime}\right)$ be labelled graphs with labels from $[d]$. A homomorphism of labelled graphs from $G$ to $G^{\prime}$ is a function $\Phi: W \rightarrow W^{\prime}$ such that for all $(v, p, w) \in R$, we have $(\Phi(v), p, \Phi(w)) \in R^{\prime}$. An $(n, d+1)$-universal graph $S$ is an even graph with labels from $[d]$ such that for all even graphs $G$ with labels from $[d]$ and with $|G| \leq n$, there is a homomorphism from $G$ to $S$.

We fix an $(n(d+1),(d+1))$-universal graph $S=(Z, K)$, noting that there are $(n(d+1),(d+1))$-universal graphs (obtained from universal trees) of size quasipolynomial in $n$ and $d$ [14]. We now combine the system $f$ with the universal graph $S$ to turn the parity conditions associated to general systems of fixpoint equations into a safety condition, associated to a single greatest fixpoint equation. 
Definition 5.2 (Chained-product fixpoint). We define a function

$$
\begin{aligned}
g: \mathcal{P}\left(B_{L} \times[k] \times Z\right) & \rightarrow \mathcal{P}\left(B_{L} \times[k] \times Z\right) \\
U & \mapsto\left\{(v, p, q) \in B_{L} \times[k] \times Z \mid v \sqsubseteq f_{p}\left(P_{0}^{U, q}, \ldots, P_{k}^{U, q}\right)\right\}
\end{aligned}
$$

where

$$
P_{i}^{U, q}=\bigsqcup\left\{u \in B_{L} \mid \exists s \in K_{\mathrm{ad}(i)}(q) .(u, i, s) \in U\right\} .
$$

We refer to $Y_{0}=$ GFP $g\left(Y_{0}\right)$ as the chained-product fixpoint (equation) of $f$ and $S$.

We now show our central result: apart from the annotation with states from the universal graph, the chained-product fixpoint $g$ is the solution of the system $f$.

Theorem 5.3. For all $u \in B_{L}$ and $0 \leq i \leq k$, we have

$u \sqsubseteq \llbracket X_{i} \rrbracket_{f}$ if and only if there is $q \in Z$ such that $(u, i, q) \in \llbracket Y_{0} \rrbracket_{g}$.

Proof. For the forward direction, let $u \sqsubseteq \llbracket X_{i} \rrbracket_{f}$. By Lemma 4.6, there is a historyfree witness $G=(W, R)$ for $u \sqsubseteq \llbracket X_{i} \rrbracket_{f}$. Since $S$ is a $(n(d+1), d+1)$-universal graph and since $G$ is a witness and hence an even labelled graph of suitable size $|G| \leq n(d+1)$, there is a graph homomorphism $\Phi$ from $G$ to $S$. Starting at $(u, i, \Phi(u, i), 0)$, we inductively construct a witness for containment of $(u, i, \Phi(u, i))$ in $\llbracket Y_{0} \rrbracket_{g}$. When at $\left(v_{1}, p_{1}, \Phi\left(v_{1}, p_{1}\right), 0\right)$ with $\left(v_{1}, p_{1}\right) \in W$, we put

$$
\begin{aligned}
R_{0}^{\prime}\left(v_{1}, p_{1}, \Phi\left(v_{1}, p_{1}\right), 0\right)= & \left\{\left(v_{2}, p_{2}, \Phi\left(v_{2}, p_{2}\right), 0\right) \in B_{L} \times[d] \times Z \times[0] \mid\right. \\
& \left.\left(v_{2}, p_{2}\right) \in R_{\mathrm{ad}\left(p_{2}\right)}\left(v_{1}, p_{1}\right), \Phi\left(v_{2}, p_{2}\right) \in K_{\mathrm{ad}\left(p_{2}\right)}\left(\Phi\left(v_{1}, p_{1}\right)\right)\right\}
\end{aligned}
$$

and continue the inductive construction with all these $\left(v_{2}, p_{2}, \Phi\left(v_{2}, p_{2}\right), 0\right)$, having $\left(v_{2}, p_{2}\right) \in W$. The resulting structure $G^{\prime}=\left(W^{\prime}, R^{\prime}\right)$ indeed is a witness for containment of $(u, i, q)$ in $\llbracket Y_{0} \rrbracket_{g}: G^{\prime}$ is even by construction. Moreover, we need to show that for $\left(v_{1}, p_{1}, \Phi\left(v_{1}, p_{1}\right), 0\right) \in W^{\prime}$, we have $\left(v_{1}, p_{1}, \Phi\left(v_{1}, p_{1}\right), 0\right) \in$ $g\left(\pi_{1}\left[R_{0}^{\prime}\left(v_{1}, p_{1}, \Phi\left(v_{1}, p_{1}\right), 0\right)\right]\right)$, i.e. $v_{1} \sqsubseteq f_{p_{1}}\left(P_{0}^{U, \Phi\left(v_{1}, p_{1}\right)}, \ldots, P_{k}^{U, \Phi\left(v_{1}, p_{1}\right)}\right)$ where $U=\pi_{1}\left[R_{0}^{\prime}\left(v_{1}, p_{1}, \Phi\left(v_{1}, p_{1}\right), 0\right)\right]$. Since $G$ is a witness and $\left(v_{1}, p_{1}\right) \in W$ by construction of $W^{\prime}$, we have $v_{1} \sqsubseteq f_{p_{1}}\left(U_{0}, \ldots, U_{k}\right)$ where $U_{j}=\bigsqcup\left(\pi_{j}\left[R_{\text {ad }(i)}\left(v_{1}, p_{1}\right)\right]\right)$. By monotonicity of $f_{p_{1}}$, it thus suffices to show that $U_{j} \sqsubseteq P_{j}^{U, \Phi\left(v_{1}, p_{1}\right)}$ for $0 \leq j \leq k$; by definition of $P_{j}^{U, \Phi\left(v_{1}, p_{1}\right)}$ this follows if

$$
\pi_{1}\left[R_{\mathrm{ad}(j)}\left(v_{1}, p_{1}\right)\right] \subseteq\left\{u \in B_{L} \mid \exists s \in K_{\mathrm{ad}(j)}\left(\Phi\left(v_{1}, p_{1}\right)\right) .(u, j, s) \in W\right\},
$$

where $W=\pi_{1}\left[R_{0}^{\prime}\left(v_{1}, p_{1}, q_{1}, 0\right)\right]$. So let $w \in B_{L}$ such that $w \in \pi_{1}\left[R_{\mathrm{ad}(j)}\left(v_{1}, p_{1}\right)\right]$. Since $R$ is a witness that is constructed as in the proof of Lemma 4.6, we have $i=\operatorname{ad}\left(i^{\prime}\right)$ for all $\left(\left(v^{\prime}, p^{\prime}\right), i,\left(w^{\prime}, i^{\prime}\right)\right) \in R$. Thus $(w, j) \in R_{\text {ad }(j)}\left(v_{1}, p_{1}\right)$ for some $j$ such that $\operatorname{ad}(j)=i$, that is, $\left(\left(v_{1}, p_{1}\right), \operatorname{ad}(j),(w, j)\right) \in R$, hence $\left(\Phi\left(v_{1}, p_{1}\right), \operatorname{ad}(j), \Phi(w, j)\right) \in K$ because $\Phi$ is a graph homomorphism. By definition of $R_{0}^{\prime}$ we have $(w, j, \Phi(w, j), 0) \in R_{0}^{\prime}\left(v_{1}, p_{1}, \Phi\left(v_{1}, p_{1}\right), 0\right)$ so that $(w, j, \Phi(w, j)) \in \pi_{1}\left[R_{0}^{\prime}\left(v_{1}, p_{1}, \Phi\left(v_{1}, p_{1}\right), 0\right)\right]$. We are done since $\Phi(w, j) \in$ $K_{\mathrm{ad}(j)}\left(\Phi\left(v_{1}, p_{1}\right)\right)$. 
For the converse implication, let $\left(u_{0}, p_{0}, q_{0}\right) \in \llbracket Y_{0} \rrbracket_{g}$ for some $q_{0} \in Z$. Let $G=(W, R)$ be a history-free witness for this fact. By Lemma 4.3, it suffices to provide a strategy in the fixpoint game for the system $f$ with which Eloise wins the node $\left(u_{0}, p_{0}\right)$. We inductively construct a history-dependent strategy $s$ as follows: For $i \geq 0$, we abbreviate $U_{i}=R_{0}\left(u_{i}, p_{i}, q_{i}, 0\right)$. We put $s\left(u_{0}, p_{0}\right)=$ $\left(P_{0}^{U_{0}, q_{0}}, \ldots, P_{k}^{U_{0}, q_{0}}\right)$. For the inductive step, let

$$
\tau=\left(u_{0}, p_{0}\right),\left(P_{0}^{U_{0}, q_{0}}, \ldots, P_{k}^{U_{0}, q_{0}}\right), \ldots,\left(P_{0}^{U_{n-1}, q_{n-1}}, \ldots, P_{k}^{U_{n-1}, q_{n-1}}\right),\left(u_{n}, p_{n}\right)
$$

be a partial play of the fixpoint game that follows the strategy that has been constructed so far. Then we have an $R$-path $\left(u_{0}, p_{0}, q_{0}, 0\right),\left(u_{1}, p_{1}, q_{1}, 0\right), \ldots,\left(u_{n}, p_{n}, q_{n}, 0\right)$, where, for $0 \leq i<n$, we have $\left(q_{i}, p_{i+1}, q_{i+1}\right) \in K$ since $u_{i+1} \sqsubseteq P_{p_{i+1}}^{U_{i}, q_{i}}$ by the inductive construction. Put $s(\tau)=\left(P_{0}^{U_{n}, q_{n}}, \ldots, P_{k}^{U_{n}, q_{n}}\right)$. Since $G$ is a witness, the strategy uses only moves that are available to Eloise (i.e. ones with $\left.u_{n} \sqsubseteq f_{p_{n}}(s(\tau))\right)$. Also, $s$ is a winning strategy as can be seen by looking at the $K$-paths that are induced by complete plays $\tau$ that follow $s$, as described (for partial plays) above. Since $S$ is a universal graph and hence even, every such $K$-path is even and the sequence of priorities in $\tau$ is just the sequence of priorities of one of these $K$-paths.

Remark 5.4. Since the set $\llbracket Y_{0} \rrbracket_{g}$ is the greatest fixpoint of $g$, it can be computed by simple approximation from above, that is, as $g^{m}\left(B_{L} \times[k] \times Z\right)$ where $m=$ $\left|B_{L} \times[k] \times Z\right|$. However, each iteration of the function $g$ may require up to $|Z|$ evaluations of an equation. In the next section, we will show how this additional iteration factor in the computation of $\llbracket Y_{0} \rrbracket_{g}$ can be avoided.

\section{A Progress Measure Algorithm}

We next introduce a lifting algorithm that computes the set $\llbracket Y_{0} \rrbracket_{g}$ efficiently, following the paradigm of the progress measure approach for parity games (e.g. $[27,28])$. Our progress measures will map pairs $(u, i) \in B_{L} \times[k]$ to nodes in a universal graph that is equipped with a simulation order, that is, a total order that is suitable for measuring progress.

Definition 6.1 (Simulation order). For natural numbers $i, i^{\prime}$, we put $i \succeq i^{\prime}$ if and only if either $i$ is even and $i=i^{\prime}$, or both $i$ and $i^{\prime}$ are odd and $i \geq i^{\prime}$. A total order $\leq$ on $Z$ is a simulation order if for all $q, q^{\prime} \in Z$,

$q \leq q^{\prime}$ implies that for all $0 \leq i \leq k$ and $s \in K_{i}(q)$, there are $i^{\prime} \succeq i$ and $s^{\prime} \in K_{i^{\prime}}\left(q^{\prime}\right)$ such that $s \leq s^{\prime}$.

Lemma 6.2. There is an $(n(d+1), d+1)$-universal graph $(Z, K)$ of size quasipolynomial in $n$ and $d$, and over which a simulation order $\leq$ exists.

Proof (Sketch). It has been shown [14, Theorem 2.2] (originally, in different terminology, [28]) that there are $(l, h)$-universal trees (a concept similar to, but 
slightly more concrete than universal graphs) with set of leaves $T$ such that $|T| \leq 2 l\left(\begin{array}{c}\log l+h+1 \\ h\end{array}\right)$. Leaves in universal trees are identified by navigation paths, that is, sequences of branching directions, so that the leaves are linearly ordered by the lexicographic order $\leq$ on navigation paths (which orders leafs from the left to the right). As described in [13], one can obtain a universal graph $(T, K)$ over $T$ in which transitions $\left(q, i, q^{\prime}\right) \in K$ for odd $i$ (the crucial case) move to the left, that is, $q^{\prime}$ is a leaf that is to the left of $q$ in the universal tree (so that $q^{\prime}<q$ ), ensuring universality. As it turns out, the lexicographic ordering on $T$ is a simulation order. Adapting this construction to our setting, we put $l=n(d+1)$ and $h=d+1$ and obtain a $(n(d+1), d+1)$-universal graph (along with a simulation order $\leq)$ of size at most $2 n(d+1)\left(\begin{array}{c}\log (n(d+1))+d+2 \\ d+1\end{array}\right)$ which is quasipolynomial in $n$ and $d$.

We fix an $(n(d+1), d+1)$-universal graph $(Z, K)$ and a simulation order $\leq$ on $Z$ for the remainder of the paper (these exist by the above lemma).

Definition 6.3 (Progress measure, lifting function). We let $q_{\min } \in Z$ denote the least node w.r.t. $\leq$ and fix a distinguished top element $\star \notin Z$, and extend $\geq$ to $Z \cup\{\star\}$ by putting $\star \geq q$ for all $q \in Z$. A measure is a map $\mu: B_{L} \times[k] \rightarrow Z \cup\{\star\}$, i.e. assigns nodes in the universal graph or $\star$ to pairs $(v, p) \in B_{L} \times[k]$. A measure $\mu$ is a progress measure if whenever $\mu(v, p) \neq \star$, then $v \sqsubseteq f_{p}\left(U_{0}^{\mu, q}, \ldots, U_{k}^{\mu, q}\right)$ where $q=\mu(v, p)$ and

$$
U_{i}^{\mu, q}=\bigsqcup\left\{u \in B_{L} \mid \exists s \in K_{\mathrm{ad}(i)}(q) \cdot \mu(u, i) \leq s\right\} .
$$

We define a function Lift $:\left(B_{L} \times[k] \rightarrow Z \cup\{\star\}\right) \rightarrow\left(B_{L} \times[k] \rightarrow Z \cup\{\star\}\right)$ on measures by

$$
(\operatorname{Lift}(\mu))(v, p)=\min \left\{q \in Z \mid v \sqsubseteq f_{p}\left(U_{0}^{\mu, q}, \ldots, U_{k}^{\mu, q}\right)\right\}
$$

where $\min \left(Z^{\prime}\right)$ denotes the least element of $Z^{\prime}$ w.r.t. $\leq$, for $\emptyset \neq Z^{\prime} \subseteq Z$; also we put $\min (\emptyset)=\star$.

The lifting algorithm then starts with the least measure $\mathrm{m}_{\min }$ that maps all pairs $(v, p) \in B_{L} \times[k]$ to the minimal node (i.e. $\mathrm{m}_{\min }(v, p)=q_{\min }$ ) and repeatedly updates the current measure using Lift until the measure stabilizes.

\section{Lifting algorithm}

(1) Initialize: Put $\mu:=\mathrm{m}_{\min }$.

(2) If $\operatorname{Lift}(\mu) \neq \mu$, then put $\mu:=\operatorname{Lift}(\mu)$ and go to 2 . Otherwise go to 3 .

(3) Return the set $\mathbb{E}=\left\{(v, p) \in B_{L} \times[k] \mid \mu(v, p) \neq \star\right\}$.

Lemma 6.4 (Correctness). For all $v \in B_{L}$ and $0 \leq p \leq k$, we have

$$
(v, p) \in \mathbb{E} \text { if and only if } v \in \llbracket X_{p} \rrbracket_{f} .
$$


Proof (Sketch). Let $\mu$ denote the progress measure that the algorithm computes. For one direction of the proof, let $(v, p) \in \mathbb{E}$. By Lemma 4.6 it suffices to construct a witness for $v \in \llbracket X_{p} \rrbracket_{f}$. We extract such a witness $(\mathbb{E}, R)$ from the progress measure $\mu$, relying on the properties of the simulation order $\leq$ that is used to measure the progress of $\mu$ to ensure that any infinite sequence of measures that $\mu$ assigns to some $R$-path induces an infinite (and hence even) path in the employed universal graph. This shows that $(\mathbb{E}, R)$ indeed is an even graph and hence a witness. For the converse direction, let $v \in \llbracket X_{p} \rrbracket_{f}$ so that there is, by Theorem 5.3, some $q \in Z$ such that $(v, p, q) \in \llbracket Y_{0} \rrbracket_{g}$. For $(u, i)$ such that there is $q^{\prime} \in Z$ such that $\left(u, i, q^{\prime}\right) \in \llbracket Y_{0} \rrbracket_{g}$, let $q_{(u, i)} \in Z$ denote the minimal such node w.r.t. $\leq$. It now suffices that $\mu(u, i) \leq q_{(u, i)}$ for all such $(u, i)$, which is shown by induction on the number of iterations of the lifting algorithm.

Corollary 6.5. Solutions of systems of fixpoint equations can be computed with quasipolynomially many evaluations of equations.

Proof. Given an $(n(d+1), d+1)$-universal graph $(Z, K)$ and a simulation order on $Z$, the lifting algorithm terminates and returns the solution of $f$ after at most $n(d+1) \cdot|Z|$ many iterations. This is the case since each iteration (except the final iteration) increases the measure for at least one of the $n(d+1)$ nodes and the measure of each node can be increased at most $|Z|$ times. Using the universal graph and the simulation order from the proof of Lemma 6.2, we have $|Z| \leq 2 n(d+1)\left(\begin{array}{c}\log (n(d+1))+d+2 \\ d+1\end{array}\right)$ so that the algorithm terminates after at most $2(n(d+1))^{2}\left(\begin{array}{c}\log (n(d+1))+d+2 \\ d+1\end{array}\right) \in \mathcal{O}\left((n(d+1))^{\log (d+1)}\right)$ iterations of the function Lift. Each iteration can be implemented to run with at most $n(d+1)$ evaluations of an equation.

Corollary 6.6. The number of function calls required for the solution of systems of fixpoint equations with $d \leq \log n$ is bounded by a polynomial in $n$ and $d$.

Proof. Following the insight of Theorem 2.8 in [9], Theorem 2.2. in [14] implies that if $d<\log n$, then there is an $(n(d+1), d+1)$-universal tree of size polynomial in $n$ and $d$. In the same way as in the proof of Lemma 6.2, one obtains a universal graph of polynomial size and a simulation order on it.

Example 6.7. Applying Corollary 6.5 and Corollary 6.6 to Example 3.2, we obtain the following results:

(1) The model checking problems for the energy $\mu$-calculus and finite latticed $\mu$-calculi are in QP. For energy parity games with sufficient upper bound $b$ on energy level accumulations, we obtain a progress measure algorithm that terminates after a number of iterations that is quasipolynomial in $b$.

(2) Under mild assumptions on the modalities (see [24]), the model checking problem for the coalgebraic $\mu$-calculus is in QP; in particular, this yields QP model checking algorithms for the graded $\mu$-calculus and the two-valued probabilistic $\mu$-calculus (equivalently: QP progress measure algorithms for solving graded and two-valued probabilistic parity games). 
(3) Under mild assumptions on the modalities (see [25]), we obtain a novel upper bound $2^{\mathcal{O}(n d \log n)}$ for the satisfiability problems of coalgebraic $\mu$-calculi, in particular including the monotone $\mu$-calculus, the alternating-time $\mu$-calculus, the graded $\mu$-calculus and the (two-valued) probabilistic $\mu$-calculus, even when the latter two are extended with (monotone) polynomial inequalities. This improves on the best previous bounds in all cases.

\section{Conclusion}

We have shown how to use universal graphs to compute solutions of systems of fixpoint equations $X_{i}=\eta_{i} \cdot f_{i}\left(X_{0}, \ldots, X_{k}\right)$ (with the $\eta_{i}$ marking least or greatest fixpoints) that use functions $f_{i}: L^{k+1} \rightarrow L$ (over a finite lattice $L$ with basis $B_{L}$ ) and involve up to $k+1$-fold nesting of fixpoints. Our progress measure algorithm needs quasipolynomially many evaluations of equations, and runs in time $\mathcal{O}(q \cdot t(f))$, where $q$ is a quasipolynomial in $\left|B_{L}\right|$ and the alternation depth of the equation system, and where $t(f)$ is an upper bound on the time it takes to compute $f_{i}$ for all $i$.

As a consequence of our results, the upper time bounds for the evaluation of various general parity conditions improve. Example domains beyond solving parity games to which our algorithm can be instantiated comprise model checking for latticed $\mu$-calculi and solving latticed parity games $[7,30]$, solving energy parity games and model checking for the energy $\mu$-calculus $[2,10]$, and model checking and satisfiability checking for the coalgebraic $\mu$-calculus [12]. The resulting model checking algorithms for latticed $\mu$-calculi and the energy $\mu$-calculus run in time quasipolynomial in the provided basis of the respective lattice. In terms of concrete instances of the coalgebraic $\mu$-calculus, we obtain, e.g., quasipolynomial-time model checking for the graded [32] and the probabilistic $\mu$-calculus $[12,34]$ as new results (corresponding results for, e.g., the alternating-time $\mu$-calculus [1] and the monotone $\mu$-calculus [18] follow as well but have already been obtained in our previous work [24]), as well as improved upper bounds for satisfiability checking in the graded $\mu$-calculus, the probabilistic $\mu$-calculus, the monotone $\mu$-calculus, and the alternating-time $\mu$-calculus. We foresee further applications, e.g. in the computation of fair bisimulations and fair equivalence $[26,31]$ beyond relational systems, e.g. for probabilistic systems.

As in the case of parity games, a natural open question that remains is whether solutions of fixpoint equations can be computed in polynomial time (which would of course imply that parity games can be solved in polynomial time). A more immediate perspective for further investigation is to generalize the recent quasipolynomial variant [38] of Zielonka's algorithm [43] for solving parity games to solving systems of fixpoint equations, with a view to improving efficiency in practice.

\section{References}

1. Alur, R., Henzinger, T., Kupferman, O.: Alternating-time temporal logic. J. ACM 49, 672-713 (2002), https://doi.org/10.1145/585265.585270 
2. Amram, G., Maoz, S., Pistiner, O., Ringert, J.O.: Energy mu-calculus: Symbolic fixed-point algorithms for omega-regular energy games. CoRR abs/2005.00641 (2020), https://arxiv.org/abs/2005.00641

3. Arnold, A., Niwinski, D., Parys, P.: A quasi-polynomial black-box algorithm for fixed point evaluation. In: Computer Science Logic, CSL 2021. LIPIcs, vol. 183, pp. 9:1-9:23. Schloss Dagstuhl - Leibniz-Zentrum für Informatik (2021), https: //doi.org/10.4230/LIPIcs.CSL.2021.9

4. Baldan, P., König, B., Mika-Michalski, C., Padoan, T.: Fixpoint games on continuous lattices. In: Principles of Programming Languages, POPL 2021. Proceedings of the ACM on Programming Languages, vol. 3, pp. 26:1-26:29. ACM (2019), https://doi.org/10.1145/3290339

5. Bodlaender, H., Dinneen, M., Khoussainov, B.: On game-theoretic models of networks. In: Algorithms and Computation, ISAAC 2001. LNCS, vol. 2223, pp. 550561. Springer (2001), https://doi.org/10.1007/3-540-45678-3_47

6. Boker, U., Lehtinen, K.: On the way to alternating weak automata. In: Foundations of Software Technology and Theoretical Computer Science, FSTTCS 2018. LIPIcs, vol. 122, pp. 21:1-21:22. Schloss Dagstuhl - Leibniz-Zentrum für Informatik (2018), https://doi.org/10.4230/LIPIcs.FSTTCS.2018.21

7. Bruns, G., Godefroid, P.: Model checking with multi-valued logics. In: Automata, Languages and Programming, ICALP 2004. LNCS, vol. 3142, pp. 281-293. Springer (2004), https://doi.org/10.1007/978-3-540-27836-8_26

8. Bruse, F., Falk, M., Lange, M.: The fixpoint-iteration algorithm for parity games. In: Games, Automata, Logics and Formal Verification, GandALF 2014. EPTCS, vol. 161, pp. 116-130. Open Publishing Association (2014), https://doi.org/10. 4204/EPTCS.161.12

9. Calude, C., Jain, S., Khoussainov, B., Li, W., Stephan, F.: Deciding parity games in quasipolynomial time. In: Theory of Computing, STOC 2017. pp. 252-263. ACM (2017), https://doi.org/10.1145/3055399.3055409

10. Chatterjee, K., Doyen, L.: Energy parity games. Theor. Comput. Sci. 458, 49-60 (2012). https://doi.org/10.1016/j.tcs.2012.07.038

11. Chatterjee, K., Dvorák, W., Henzinger, M., Svozil, A.: Quasipolynomial set-based symbolic algorithms for parity games. In: Logic for Programming, Artificial Intelligence and Reasoning, LPAR 2018. EPiC, vol. 57, pp. 233-253. EasyChair (2018), https://doi.org/10.29007/5z5k

12. Cîrstea, C., Kupke, C., Pattinson, D.: EXPTIME tableaux for the coalgebraic $\mu$ calculus. Log. Meth. Comput. Sci. 7 (2011), https://doi.org/10.2168/LMCS-7(3: 3) 2011

13. Colcombet, T., Fijalkow, N.: Universal graphs and good for games automata: New tools for infinite duration games. In: Foundations of Software Science and Computation Structures, FOSSACS 2019. LNCS, vol. 11425, pp. 1-26. Springer (2019), https://doi.org/10.1007/978-3-030-17127-8_1

14. Czerwinski, W., Daviaud, L., Fijalkow, N., Jurdzinski, M., Lazic, R., Parys, P.: Universal trees grow inside separating automata: Quasi-polynomial lower bounds for parity games. In: Symposium on Discrete Algorithms, SODA 2019. pp. 23332349. SIAM (2019), https://doi.org/10.1137/1.9781611975482.142

15. Daviaud, L., Jurdzinski, M., Lazic, R.: A pseudo-quasi-polynomial algorithm for mean-payoff parity games. In: Logic in Computer Science, LICS 2018. pp. 325-334. ACM (2018), https://doi.org/10.1145/3209108.3209162

16. Dawar, A., Grädel, E.: The descriptive complexity of parity games. In: Computer Science Logic, CSL 2008. LNCS, vol. 5213, pp. 354-368. Springer (2008), https: //doi.org/10.1007/978-3-540-87531-4_26 
17. Emerson, E.A., Jutla, C., Sistla, A.P.: On model checking for the $\mu$-calculus and its fragments. Theor. Comput. Sci. 258, 491-522 (2001), https://doi.org/10.1016/ S0304-3975(00)00034-7

18. Enqvist, S., Seifan, F., Venema, Y.: Monadic second-order logic and bisimulation invariance for coalgebras. In: Logic in Computer Science, LICS 2015. pp. 353-365. IEEE (2015), https://doi.org/10.1109/LICS.2015.41

19. Fearnley, J., Jain, S., de Keijzer, B., Schewe, S., Stephan, F., Wojtczak, D.: An ordered approach to solving parity games in quasi-polynomial time and quasi-linear space. STTT 21(3), 325-349 (2019), https://doi.org/10.1007/s10009-019-00509-3

20. Friedmann, O., Lange, M.: Deciding the unguarded modal $\mu$-calculus. J. Appl. NonClassical Log. 23, 353-371 (2013), https://doi.org/10.1080/11663081.2013.861181

21. Grädel, E., Thomas, W., Wilke, T. (eds.): Automata, Logics, and Infinite Games: A Guide to Current Research, LNCS, vol. 2500. Springer (2002), https://doi.org/ 10.1007/3-540-36387-4

22. Hasuo, I., Shimizu, S., Cîrstea, C.: Lattice-theoretic progress measures and coalgebraic model checking. In: Principles of Programming Languages, POPL 2016. pp. 718-732. ACM (2016), https://doi.org/10.1145/2837614.2837673

23. Hausmann, D., Schröder, L.: Computing nested fixpoints in quasipolynomial time. CoRR abs/1907.07020 (2019), http://arxiv.org/abs/1907.07020

24. Hausmann, D., Schröder, L.: Game-based local model checking for the coalgebraic $\mu$-calculus. In: Concurrency Theory, CONCUR 2019. LIPIcs, vol. 140, pp. 35:135:16. Schloss Dagstuhl-Leibniz-Zentrum fuer Informatik (2019), https://doi.org/ 10.4230/LIPIcs.CONCUR.2019.35

25. Hausmann, D., Schröder, L.: Optimal satisfiability checking for arithmetic $\mu$ calculi. In: Foundations of Software Science and Computation Structures, FOSSACS 2019. LNCS, vol. 11425, pp. 277-294. Springer (2019), https://doi.org/10. 1007/978-3-030-17127-8_16

26. Henzinger, T., Rajamani, S.: Fair bisimulation. In: Tools and Algorithms for Construction and Analysis of Systems, TACAS 2000. LNCS, vol. 1785, pp. 299-314. Springer (2000), https://doi.org/10.1007/3-540-46419-0_21

27. Jurdziński, M.: Small progress measures for solving parity games. In: Symposium on Theoretical Aspects of Computer Science, STACS 2000. LNCS, vol. 1770, pp. 290-301. Springer (2000), https://doi.org/10.1007/3-540-46541-3_24

28. Jurdzinski, M., Lazic, R.: Succinct progress measures for solving parity games. In: Logic in Computer Science, LICS 2017. pp. 1-9. IEEE Computer Society (2017), https://doi.org/10.1109/LICS.2017.8005092

29. Kozen, D.: Results on the propositional $\mu$-calculus. Theor. Comput. Sci. 27, 333354 (1983), https://doi.org/10.1016/0304-3975(82)90125-6

30. Kupferman, O., Lustig, Y.: Latticed simulation relations and games. In: Automated Technology for Verification and Analysis, ATVA 2007. LNCS, vol. 4762, pp. 316330. Springer (2007), https://doi.org/10.1007/978-3-540-75596-8_23

31. Kupferman, O., Piterman, N., Vardi, M.: Fair equivalence relations. In: Verification: Theory and Practice. LNCS, vol. 2772, pp. 702-732. Springer (2003), https://doi.org/10.1007/978-3-540-39910-0_30

32. Kupferman, O., Sattler, U., Vardi, M.: The complexity of the graded $\mu$-calculus. In: Automated Deduction, CADE 2002. LNCS, vol. 2392, pp. 423-437. Springer (2002), https://doi.org/10.1007/3-540-45620-1_34

33. Lehtinen, K.: A modal $\mu$ perspective on solving parity games in quasi-polynomial time. In: Logic in Computer Science, LICS 2018. pp. 639-648. ACM (2018), https: //doi.org/10.1145/3209108.3209115 
34. Liu, W., Song, L., Wang, J., Zhang, L.: A simple probabilistic extension of modal mu-calculus. In: International Joint Conference on Artificial Intelligence, IJCAI 2015. pp. 882-888. AAAI Press (2015), http://ijcai.org/proceedings/2015

35. Long, D.E., Browne, A., Clarke, E.M., Jha, S., Marrero, W.R.: An improved algorithm for the evaluation of fixpoint expressions. In: Computer Aided Verification, CAV 1994. LNCS, vol. 818, pp. 338-350. Springer (1994), https://doi.org/10.1007/ 3-540-58179-0_66

36. Mio, M.: On the equivalence of game and denotational semantics for the probabilistic $\mu$-calculus. Log. Methods Comput. Sci. 8 (2012), https://doi.org/10.2168/ LMCS-8(2:7)2012

37. Parikh, R.: The logic of games and its applications. Ann. Discr. Math. 24, 111-140 (1985), https://doi.org/10.1016/S0304-0208(08)73078-0

38. Parys, P.: Parity games: Zielonka's algorithm in quasi-polynomial time. In: Mathematical Foundations of Computer Science, MFCS 2019. LIPIcs, vol. 138, pp. 10:1-10:13. Schloss Dagstuhl - Leibniz-Zentrum für Informatik (2019), https: //doi.org/10.4230/LIPIcs.MFCS.2019.10

39. Peleg, D.: Concurrent dynamic logic. J. ACM 34, 450-479 (1987), https://doi.org/ $10.1145 / 23005.23008$

40. Rutten, J.: Universal coalgebra: A theory of systems. Theor. Comput. Sci. 249, 3-80 (2000), https://doi.org/10.1016/S0304-3975(00)00056-6

41. Seidl, H.: Fast and Simple Nested Fixpoints. Inf. Process. Lett. 59, 303-308 (1996), https://doi.org/10.1016/0020-0190(96)00130-5

42. Venema, Y.: Lectures on the modal $\mu$-calculus. Lecture notes, Institute for Logic, Language and Computation, Universiteit van Amsterdam (2008), https://staff. fnwi.uva.nl/y.venema/teaching/ml/notes/20201212-mu.pdf

43. Zielonka, W.: Infinite games on finitely coloured graphs with applications to automata on infinite trees. Theor. Comput. Sci. 200(1-2), 135-183 (1998), https: //doi.org/10.1016/S0304-3975(98)00009-7

Open Access This chapter is licensed under the terms of the Creative Commons Attribution 4.0 International License (https://creativecommons.org/licenses/by/4.0/), which permits use, sharing, adaptation, distribution and reproduction in any medium or format, as long as you give appropriate credit to the original author(s) and the source, provide a link to the Creative Commons license and indicate if changes were made.

The images or other third party material in this chapter are included in the chapter's Creative Commons license, unless indicated otherwise in a credit line to the material. If material is not included in the chapter's Creative Commons license and your intended use is not permitted by statutory regulation or exceeds the permitted use, you will need to obtain permission directly from the copyright holder.

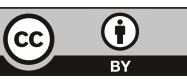

